\title{
Privatisation des sociétés d'autoroute et marché aval $*$
}

\author{
David Ettinger ${ }^{\dagger}$
}

17 novembre 2008

\begin{abstract}
Résumé
Nous analysons les privatisations des sociétés d'autoroute en nous intéressant à la fois à la vente des actions de ces sociétés et aux appels d'offre qu'elles organisent pour réaliser l'entretien du réseau après la vente des actions. Nous expliquons l'intérêt des entreprises de travaux publics pour les actions des sociétés d'autoroute par la possibilité qu'elles leurs offrent de peser indirectement sur les appels d'offre en aval, tout en respectant les règles de non-discrimination. Si une entreprise de travaux publics achète ces actions, les appels d'offre deviennent inefficaces et le revenu des sociétés d'autoroute augmente. Or,l'utilisation d'une enchère standard pour vendre ces actions aboutit précisément à un tel résultat. Nous proposons un mécanisme d'allocation des actions des sociétés d'autoroute qui maximise le revenu de l'Etat et restaure l'efficacité des appels d'offres en aval.
\end{abstract}

\section{Introduction}

En 2002, le gouvernement français décide de privatiser 49,6\% de la société d'autoroute ASF (Autoroutes du Sud de la France, 2,47 milliards d'euros de chiffre d'affaires en 2005 et $3.124 \mathrm{~km}$ de réseau en concession). Cette première opération est suivie par les privatisations partielles des sociétés APRR (Autoroutes Paris-Rhin-Rhône, 1,57 milliards d'euros de chiffre d'affaires en 2005 et $2.260 \mathrm{~km}$ de réseau en concession ${ }^{1}$ ), en 2004, à hauteur de 30\% du capital et SANEF (Société des Autoroutes du Nord et

*Je voudrais remercier Philippe Jéhiel, Anne Perrot, Jean-Christophe Vergnaud, Shmuel Zamir, un rapporteur anonyme ainsi que les participants aux séminaires du CREST-LEI et du groupe de travail en Microéconomie du THEMA pour leurs précieux commentaires et soutien.

†THEMA, UMR 8184, Université de Cergy-Pontoise, 33 boulevard du Port, 95011 Cergy-Pontoise cedex, France; ettinger@u-cergy.fr.

${ }^{1}$ En incluant sa filiale à $99,82 \%$, AREA. 
de l'Est de la France, 1,15 milliard d'euros de chiffre d'affaires en 2005 et $1.772 \mathrm{~km}$ de réseau en concession), au printemps 2005 , à hauteur de $26 \%$ du capital ${ }^{2}$.

Le 18 juillet 2005, le nouveau gouvernement français décide d'entamer une seconde vague de privatisations de ces sociétés d'autoroute et de vendre la plus grande partie des actions de ces sociétés qu'il détient encore ${ }^{3}$. L'objectif affiché du gouvernement est d'utiliser les fonds obtenus pour le désendettement de l'Etat et le financement d'investissements porteurs d'avenir pour notre économie.

Parmi les principales sociétés ayant fait des propositions pour l'achat de ces actions, on trouve des sociétés étrangères spécialistes du secteur ${ }^{4}$, des investisseurs financiers français et des grandes entreprises françaises de travaux publics (Vinci pour ASF et Eiffage pour APRR, concernant SANEF, le groupe Bouygues s'est finalement retiré alors qu'il considérait le dossier ${ }^{5}$ ). On remarquera, à ce propos, qu'au cours de la première vague de privatisation, Vinci avait déjà obtenu $23 \%$ d'ASF $^{6}$.

Les résultats des ventes ont été les suivants. Le groupe Vinci a effectivement obtenu les actions d'ASF mises en vente. Eiffage, associé au fond d'investissement canadien Macquarie Infrastructures, a obtenu les actions d'APRR. Enfin, les actions SANEF ont été vendues au holding HIT mené par le gestionnaire d'infrastructures espagnol Abertis adossé à des groupes financiers français tels que la Caisse des Dépôts et Consignations, Axa, Predica et la $\mathrm{CNP}^{7}$.

On peut légitimement s'interroger sur l'intérêt manifesté par les entreprises de travaux publics pour le rachat des sociétés d'autoroute. En effet, les deux activités, travaux publics et gestion d'autoroutes même si elles partagent des intérêts, sont notablement différentes. Par ailleurs, même si les entreprises de travaux publics considérées sont de tailles importantes, les marges sont traditionnellement limitées dans ce secteur d'activité. Les entreprises de travaux publics ne disposent donc pas de sommes disponibles considérables. Or, le gouvernement a exigé que le paiement s'effectue en numéraire. Les entreprises de travaux publics ont donc dû s'endetter pour financer ces investissements, accroissant leurs taux d'endettement au risque de diminuer ainsi les notes que leurs attribuent les agences de notation (des messages ont été envoyés en ce sens par ces

\footnotetext{
${ }^{2}$ Pour plus de détails, voir les articles de de Bati-Actu [2005] et de L'Express [2005] cités en annexe sur les privatisations et ces sociétés.

${ }^{3}$ Il convient de rappeler que, dans tous les cas considérés, seul le capital des sociétés gestionnaires des autoroutes a été vendu car l'Etat reste le propriétaire des infrastructures.

${ }^{4}$ On pourra citer les entreprises espagnoles Itinere, Cintra ou Abertis et l'italien Autostrade.

${ }^{5}$ Sur le retrait de Bouygues, on pourra se référer à l'article du Monde [2005] cité dans la bibliographie.

${ }^{6}$ Vinci contrôle aussi Cofiroute dont une fraction du capital était détenue par Eiffage. Eiffage a revendu en 2007 cette fraction du capital à Vinci.

${ }^{7}$ En raison de la récente doctrine du patriotisme économique, il était quasiment inenvisageable pour une entreprise étrangère d'obtenir ces actions sans s'associer à des capitaux français. D'où le choix d'Abertis de s'adosser à des entreprises françaises
} 
agences) ou s'associer à des investisseurs financiers ${ }^{8}$. On peut alors s'interroger sur les motivations des entreprises de travaux publics qui tendent à devenir des spécialistes de la gestion des concessions d'infrastructure (gestion de parkings, autoroutes, ponts, installations sportives...).

L'intérêt des entreprises de travaux publics pour les sociétés d'autoroute, malgré ces inconvénients, s'expliquerait par le fait que les entreprises de travaux publics sont les principaux fournisseurs des sociétés d'autoroute. Elles espéraient ainsi tirer un avantage direct de ces participations. Une entreprise de travaux publics, en devenant actionnaire d'une société d'autoroute, chercherait à obtenir des conditions plus avantageuses lors des appels d'offre organisés par cette société d'autoroute.

Mais ces motivations, trop naturelles pour rester méconnues, ont été anticipées par le gouvernement. Celui-ci a veillé à ce que ces conditions avantageuses ne soient pas offertes. Il a précisé, dans le cahier des charges portant sur le fonctionnement des sociétés d'autoroute après la privatisation, que l'attribution des travaux devra s'inscrire dans la transparence et la non-discrimination.

Ces mesures gouvernementales sont supposées faire disparaître les motivations spécifiques des entreprises de travaux publics. Les résultats de ces ventes indiquent cependant que ce ne fut pas le cas.

Pour comprendre les comportements des entreprises de travaux publics, nous suggérons d'autres motivations spécifiques qui pourraient expliquer la volonté de ces entreprises d'acheter des actions des sociétés d'autoroute malgré les dispositions gouvernementales.

Pour cela, nous considérons un modèle simplifié avec une seule société d'autoroute en vente qui, après que la structure de son capital est modifiée, organise un appel d'offre pour l'entretien et le renouvellement de sa voirie. Nous observons qu'une entreprise de travaux publics, si elle est actionnaire de la société d'autoroute, voit son comportement se modifier lors de l'appel d'offre. Du fait de son double statut de fournisseur potentiel et de propriétaire (partiel), il est dans l'intérêt de l'entreprise de travaux publics de soumettre une offre plus faible pour la réalisation des travaux. Cette soumission plus faible pèse à la baisse sur le prix de la réalisation du chantier et ceci non seulement quand la société de travaux publics actionnaire emporte le marché mais aussi lorsqu'un concurrent l'emporte, ce qui est dans l'intérêt de l'entreprise de travaux publics actionnaire.

Ainsi, en ayant un comportement lors des appels d'offre visant uniquement à maximiser son profit, sans manipulation du processus d'appel d'offre ni distorsion, l'actionnaire fournisseur obtient des meilleures conditions tarifaires pour la réalisation des travaux de la société d'autoroute que n'obtiendrait un actionnaire financier. Les actions des sociétés d'autoroute ont donc une valeur plus élevée pour une entreprise de

\footnotetext{
${ }^{8}$ Choix effectué par Eiffage.
} 
travaux publics que pour un investisseur financier.

Partant de cette observation initiale, nous nous intéressons au surcroît de profit espéré qu'une entreprise de travaux publics obtient en achetant des actions d'une société d'autoroute, par rapport à ce qu'obtiendrait une structure financière, du fait de son statut de fournisseur potentiel. Nous montrons qu'il est d'autant plus élevé que la structure de coût de la société de travaux publics est, a priori, défavorable et qu'elle a une probabilité faible d'emporter l'appel d'offre. La détention des actions renforce une entreprise de travaux publics faible alors qu'une entreprise de travaux publics forte a moins l'usage de cette aide.

A l'aide de cette grille de lecture portant sur les appels d'offre en aval, nous étudions la forme que prend l'allocation des actions des sociétés d'autoroute en amont.

La première particularité de cet environnement est que les entreprises candidates à l'achat des actions des sociétés d'autoroute n'ont pas de valorisations définies pour ces actions. Lorsqu'une entreprise de travaux publics s'interroge sur le montant qu'elle est disposée à payer pour acheter ces actions, elle doit aussi s'interroger sur l'identité de l'acheteur de ces actions si elle ne les achète pas elle même. En effet, si ces actions sont achetées par une entreprise concurrente, alors ce concurrent se montrera plus agressif dans les appels d'offre à venir, ce qui a un coût pour l'entreprise de travaux publics. En revanche, si les actions sont achetées par un investisseur financier, cela n'affectera pas les appels d'offre organisés par la suite. Les soumissions d'équilibre doivent alors intégrer les croyances sur l'identité du vainqueur, en cas de défaite.

Dans ce cadre, nous montrons que si les actions de la société d'autoroute sont vendues par le biais d'une enchère standard (enchère ascendante, descendante, enchère au premier prix ou second prix), une entreprise de travaux publics obtiendra les actions. Les entreprises de travaux publics ayant des motivations particulières, elles peuvent surenchérir sur un investisseur financier. Plus spécifiquement, c'est l'entreprise de travaux publics ayant la structure de coût la plus favorable pour l'appel d'offre aval qui obtiendra les actions.

Or, nous avons montré que l'entreprise de travaux publics ayant la structure de coûts la plus défavorable peut tirer le plus grand parti de la possession de ces actions. Cet apparent paradoxe s'explique non par ce que les entreprises souhaitent obtenir mais par ce qu'elles souhaitent éviter. Certes, une entreprise de travaux publics ayant une structure de coûts, a priori, plus favorable tire un moindre profit de la détention des actions de la société d'autoroute. Mais elle pâtirait fortement de l'achat de ces actions par un concurrent ayant une structure de coût défavorable. De ce fait, elle sera disposée à faire une offre élevée afin d'éviter que cette éventualité ne se réalise. C'est donc une entreprise de travaux publics qui obtiendra les actions si elles sont vendues au travers d'une enchère standard. L'allocation des travaux d'entretien de l'autoroute sera alors inefficace puisqu'un des participants à l'appel d'offre pour ces travaux est actionnaire 
de la société d'autoroute.

Dans la suite, suivant une démarche plus normative, nous proposons un mécanisme de vente des actions des sociétés d'autoroute permettant à la fois de maximiser le revenu du vendeur et de rétablir l'efficacité lors des appels d'offre pour la réalisation des travaux d'entretien. Ce mécanisme prend la forme suivante. Il consiste à allouer les actions de la société d'autoroute à un investisseur financier à qui on fait payer la valeur espérée de ces actions pour lui. Parallèlement, les entreprises de travaux publics versent un montant égal au prix qu'elles sont disposées à payer pour éviter que leur concurrent n'obtienne les actions. Ce mécanisme d'allocation est maintenu à l'équilibre par la menace pesant sur chaque entreprise de travaux publics d'allouer les actions à l'entreprise de travaux publics concurrente dans le cas où elle refuserait de verser le montant qui lui est demandé. Un tel mécanisme assure à la fois le revenu le plus élevé possible pour l'Etat et l'efficacité pour l'appel d'offre qu'organise la société d'autoroute par la suite.

Les résultats que nous obtenons sont reliés à deux séries de travaux existants. Pour ce qui concerne les appels d'offre en aval, après la vente des actions, notre démarche se rapproche de celle de BuRKart [1995], Singh [1998] ou EtTinger [2008] et dans une moindre mesure de BuLow et AL [1999]. Ils considèrent tous l'impact d'une participation d'un enchérisseur au capital du vendeur dans une enchère, ce qui s'apparente au cas que nous étudions. Nos travaux se distinguent de ceux existants par la comparaison effectuée entre les différentes allocations possibles des actions auprès des entreprises de travaux publics ayant des structures de coût plus ou moins favorable et par l'analyse d'efficacité jointe. De plus, nos travaux permettent une mise en perspective plus générale par l'intégration dans notre modélisation de la phase initiale d'allocation des actions.

Pour ce qui concerne cette phase initiale, nous nous situons dans le cadre des enchères avec externalités allocatives, cadre initié par les travaux de Jehiel et Moldovanu (voir notamment Jehiel et Moldovanu [1996a], Jehiel et AL [1996b] ou CAILlaud et JEHIEL [1998]). Notre contribution par rapport à cette littérature concerne plus spécifiquement la justification de la présence des externalités allocatives et l'emploi dans un cadre appliqué spécifique de résultats plus généraux. Ainsi, le mécanisme présenté dans la section 4 permettant la maximisation du revenu de l'Etat est une application des résultats de JEHIEL et AL [1996b].

La suite du papier est structurée de la façon suivante. La section 2 contient la présentation du modèle. La section 3 porte sur l'appel d'offre organisé par la société d'autoroute après la vente de ses actions. Enfin, la section 4 analyse la vente des actions de la société d'autoroute par l'Etat et la section 5 conclut. 


\section{Modèle}

Soit un Etat détenant une fraction $\theta \in] 0 ; 1]$ du capital d'une société d'autoroute. Cet Etat fait face à des contraintes de financement et décide de vendre un fraction $\alpha \in] 0 ; \theta]$ du capital de cette société ${ }^{9}$. Trois acheteurs sont intéressés par l'achat de cette fraction du capital de la société d'autoroute, les entreprises $A, B$ et $C$, toutes neutres au risque. Les entreprises $A$ et $B$ sont des entreprises du secteur des travaux publics et l'entreprise $C$ est un investisseur financier.

Par souci de simplicité, nous décomposons l'activité de la société d'autoroute en deux parties et considérons qu'elle n'a une durée de vie égale qu'à une seule période d'activité ${ }^{10}$. La société d'autoroute a une activité d'exploitation regroupant entre autres éléments, la collecte des péages, les redevances des stations essence, le fonctionnement des péages et le remboursement des emprunts. Par ailleurs, la société d'autoroute effectue des travaux d'entretien, de modifications et d'agrandissements du réseau autoroutier. Nous regrouperons ces activités sous le terme de travaux d'entretien. L'activité d'exploitation fournit un revenu net, $R N$, dont la valeur est connaissance commune. Les activités d'entretien ne sont pas effectuées par la société d'autoroute. Elle les soustraite à une entreprise de travaux publics et procède pour ce marché à un appel d'offre mettant en concurrence les sociétés $A$ et $B^{11}$.

L'ordonnancement des opérations que nous venons de décrire s'effectue de la façon suivante.

A la date $t=1$, l'Etat vend la participation $\alpha$ à l'une des trois entreprises au travers d'un mécanisme à définir.

A la date $t=2$, les entreprises $A$ et $B$ découvrent quels sont leurs coûts respectifs, $v_{A}$ et $v_{B}$, pour réaliser le chantier d'entretien pour lequel la société d'autoroute va les mettre en concurrence. Les valeurs de $v_{A}$ et $v_{B}$ sont des informations privées respectivement de $A$ et de $B$. En revanche, il est connaissance commune de tous les acteurs, dés la date $t=1$, que $v_{i}$, avec $i=A, B$, est extrait suivant une fonction de distribution uniforme $^{12}$ sur $\left[\underline{v}_{i} ; 1\right]$. Nous n'imposons pas l'égalité entre $\underline{v}_{A}$ et $\underline{v}_{B}$ afin de pouvoir analyser une situation où les deux entreprises de travaux publics ont des structures des

\footnotetext{
${ }^{9}$ Nous ne faisons pas de différence entres les cas $\alpha<1 / 2$ et $\alpha \geq 1 / 2$ dans la mesure où nous considérons que, du fait des dispositions prises par le gouvernement, une entreprise de travaux publics, même si elle est actionnaire majoritaire, ne peut pas distordre les appels d'offre de la société d'autoroute.

${ }^{10}$ Considérer une société d'autoroute dont l'activité s'étendrait sur plusieurs périodes ne modifierait pas l'analyse et alourdirait la présentation des résultats.

${ }^{11}$ Ajouter des participants à l'appel d'offre ne modifie pas qualitativement les résultats.

${ }^{12}$ Nous faisons le choix simplificateur d'une fonction uniforme car il permet de traiter l'asymétrie entre les deux entreprises. Cela ne pourrait s'effectuer, pour des raisons techniques, dans le cadre général. Néanmoins, l'intuition de nos résultats ne repose en rien sur la forme spécifique de ces fonctions de distribution.
} 
coûts différentes. Sans perte de généralité, nous supposons que $0=\underline{v}_{A} \leq \underline{v}_{B}<1$. Nous interprétons cette hypothèse en supposant que l'entreprise $A$ est de taille supérieure à $B$ et qu'elle dispose d'une structure de coût, en moyenne, plus favorable.

A la date $t=3$, la société d'autoroute organise un appel d'offre pour la réalisation des travaux d'entretien. Les sociétés $A$ et $B$ sont les deux seuls compétiteurs. La mise en concurrence s'effectue au travers d'un appel d'offre au second prix ${ }^{13}$.

L'appel d'offre au second prix fonctionne de la façon suivante. Les entreprises soumettent simultanément des montants pour la réalisation du chantier. L'entreprise ayant soumis le montant le plus faible est choisie pour réaliser les travaux d'entretien. Celle-ci est payée non pas le montant qu'elle a soumis mais le second montant le plus faible parmi tous ceux soumis (dans le cas présent, avec deux entreprises participantes, cela correspond au montant soumis par le concurrent). Lorsque plusieurs entreprises soumettent le même montant le plus faible, on procède à un tirage au sort équitable pour choisir le fournisseur gagnant qui sera payé le montant de la soumission commune.

A la date $t=4$, la société d'autoroute gère son activité. Elle encaisse le solde d'exploitation $R N$, paye à l'entreprise de travaux publics ayant emporté l'appel d'offre le montant prévu pour la réalisation des travaux. Cette même entreprise réalise les travaux d'entretien. Enfin, la société d'autoroute distribue ses bénéfices aux actionnaires au pro rata de leurs participations, au travers de dividendes.

Nous utilisons les notations suivantes. Soient $p_{\alpha}$ le prix payé à l'Etat pour acheter la fraction $\alpha$ du capital de la société d'autoroute mise en vente, $p_{e}$ le prix payé par la société d'autoroute pour faire réaliser l'entretien de son réseau, $X_{i}^{\alpha}$ la variable indicatrice égale à 1 si l'entreprise $i$ achète la fraction $\alpha$ du capital en vente et à 0 sinon et $X_{i}^{e}$ la variable indicatrice égale à 1 si l'entreprise $i$ est choisie pour réaliser l'entretien des routes et et à 0 sinon.

Pour mesurer l'inefficacité d'une procédure d'allocation des travaux d'entretien, nous introduisons $P S$, la perte de surplus d'une allocation égale à : $X_{A}^{e} v_{A}+X 1_{B}^{e} v_{B}-$ $\min \left(v_{A} ; v_{B}\right)$. Enfin, $P S E$ est la valeur espérée de $P S$. L'espérance est calculée par rapport aux fonctions de distribution de $v_{A}$ et $v_{B}$ et par rapport aux fonctions de soumission d'équilibre lors de l'appel d'offre pour l'allocation des travaux.

Notons que, dans notre modèle, en $t=1$, tous les acteurs disposent de la même information. L'asymétrie d'information n'apparaît que plus tardivement. Cette hypothèse ne semble pas abusive dans le cas considéré. En effet, toutes les entreprises intéressées

\footnotetext{
${ }^{13}$ Certes, l'ensemble des appels d'offre ne prend pas la forme d'une enchère au second prix (ou ascendante qui est identique dans ce cadre). Néanmoins, nous avons privilégié l'étude de ce format d'enchère pour des contraintes techniques. La double asymétrie : distribution des $v_{i}$ et détention de la participation n'est pas analysable dans le cadre de l'enchère au premier prix. Par ailleurs, Engelbrecht Wiggans [1994] et Ettinger [2002] ont montré, dans un cadre proche, que des participations affectent de façon qualitativement identique l'enchère au premier prix et l'enchère au second prix même si l'enchère au premier prix est plus faiblement affectée.
} 
par l'achat des actions disposent des mêmes informations délivrées sur la société d'autoroute. Par ailleurs, au moment où les actions sont vendues, les futurs appels d'offre sont lointains et peu définis. Par conséquent, aucune entreprise ne connaît, à ce moment, son coût à réaliser ces futurs travaux. En revanche, il peut déjà être connaissance commune qu'une entreprise dispose d'une structure de coûts ou d'une installation géographique plus favorable pour le type de travaux qui pourront être requis.

Enfin les utilités et revenus s'obtiennent de la façon suivante (avec un taux d'actualisation nulle).

Le revenu de l'Etat est égal à : $p_{\alpha}+(\theta-\alpha)\left(R N-p_{e}\right)$. Pour $i=A, B$, le profit de l'entreprise $i$ vaut : $X_{i}^{\alpha}\left[\alpha\left(R N-p_{e}\right)-p_{\alpha}\right]+X_{i}^{e}\left(p_{e}-v_{i}\right)$. Enfin, le profit de l'entreprise $C$ s'écrit : $X_{C}^{\alpha}\left[\alpha\left(R N-p_{e}\right)-p_{\alpha}\right]$.

Au cours de l'analyse, nous ne nous intéresserons qu'aux équilibres en stratégies pures non dominées. Toutes les preuves non exposées dans le corps du document se situent en annexe.

\section{L'appel d'offre pour l'entretien des routes}

Dans cette section, nous analysons les équilibres du sous-jeu constitué par l'appel d'offre pour l'entretien des routes en fonction de l'identité du vainqueur de l'enchère pour la vente de la participation $\alpha$.

En effet, parmi les quatre périodes que nous avons exposées dans la description du modèle, il n'y en a que deux au cours desquelles les entreprises ont des décisions à prendre, la première et la troisième. Lors des deux autres périodes, le déroulement des opérations est soit une conséquence des choix précédents soit subi. Nous concentrerons donc notre analyse sur les périodes 1 et 3 .

Nous commencerons par analyser le comportement des entreprises en $t=3$, conformément au mode de résolution par récurrence inverse. Nous distinguons les trois cas possibles d'allocation des actions. Pour tous ces cas, nous étudions les soumissions d'équilibre et les propriétés (revenu et efficacité) de ces équilibres.

Premier cas : l'entreprise $C$ obtient la fraction $\alpha$ du capital de la société d'autoroute en vente.

Dans ce cas, les entreprises $A$ et $B$ sont dans une situation classique d'appel d'offre. La vente de la participation au capital n'a pas d'effet sur les motivations des entreprises $A$ et $B$. Celles-ci ont une stratégie dominante : soumettre au cours de l'appel d'offre leurs véritables coûts à réaliser l'entretien du réseau autoroutier. Soumettre moins que cette valeur ne modifierait l'allocation des travaux d'entretien qu'en permettant de les obtenir pour des niveaux des prix pour lesquels il n'est pas intéressant d'obtenir leurs réalisations. Symétriquement, soumettre plus que cette valeur ferait perdre des 
occasions d'emporter le contrat de réalisation des travaux dans des situations où celui-ci serait profitable.

Proposition 1 Si l'entreprise $C$ obtient la fraction $\alpha$ du capital de la société d'autoroute, en $t=3$, il existe un unique équilibre en stratégies non dominées où les entreprises $A$ et $B$ soumettent leurs véritables coûts à réaliser l'entretien du réseau. L'allocation des travaux d'entretien est efficace. Le coût espéré des travaux d'entretien pour la société d'autoroute est : $\frac{1}{6}\left(4+\underline{v}_{B}+\underline{v}_{B}^{2}\right)$.

Chacune des entreprises soumettant son coût à réaliser les travaux, l'allocation est efficace. Notons aussi que si le gouvernement décide de ne pas vendre les actions, l'équilibre de l'appel d'offre est identique à celui décrit dans le cas où l'entreprise $C$ obtient les actions de la société d'autoroute.

Deuxième cas : l'entreprise $A$ obtient la fraction $\alpha$ du capital de la société d'autoroute en vente.

Il convient alors de distinguer la situation des deux entreprises A et B. En effet, cette modification de la structure du capital n'affecte pas les motivations de soumission de l'entreprise $B$ lors de l'appel d'offre. Pour elle, soumettre son coût à réaliser les travaux d'entretien demeure une stratégie dominante, les motivations que nous avons présentées précédemment demeurent vérifiées.

Il n'en est pas de même pour l'entreprise $A$. En effet, soumettre sa valeur n'est une stratégie dominante que dans la mesure où un enchérisseur ne se soucie pas du montant versé à son concurrent si celui-ci obtient le marché. Hors, cette hypothèse, le plus généralement vérifiée, ne l'est pas dans le cas présent. L'entreprise $A$ possédant une fraction du capital de la société d'autoroute, lorsqu'elle perd l'appel d'offre, elle préfère que l'entreprise $B$ obtienne le marché pour un prix faible (ce qui accroît les profits de la société d'autoroute). L'entreprise $A$ a donc une incitation spécifique à soumettre à un niveau plus faible pour réduire le prix payé à l'entreprise $B$. Ceci se concrétise de la façon suivante.

Proposition 2 Si l'entreprise A obtient la fraction $\alpha$ du capital de la société d'autoroute, en $t=3$, tout équilibre en stratégies non dominées prend la forme suivante. L'entreprise $B$ soumet $v_{B}$, son coût à réaliser les travaux. Si $v_{A}>\underline{v}_{B}$, l'entreprise $A$ soumet $\frac{v_{A}+\alpha \underline{v}_{B}}{1+\alpha}<v_{A}$ et si $v_{A} \leq \underline{v}_{B}$, l'entreprise $A$ soumet une offre inférieure ou égale à son coût à réaliser les travaux.

La proposition 2 se prouve de façon directe. La stratégie de l'entreprise $B$ étant fixée (stratégie dominante de soumettre son coût effectif), celle de l'entreprise $A$ s'obtient comme le résultat d'un programme de maximisation.

Considérons de façon plus précise la stratégie de l'entreprise $A$. Tant que son coût pour réaliser les travaux d'entretien est supérieur à $\underline{v}_{B}$, elle soumet moins que son coût 
à réaliser les travaux. Ce comportement s'explique par la motivation spécifique que nous avons exprimé précédemment, la volonté dans le cas où l'entreprise $B$ obtiendrait ce contrat de fixer le prix payé au niveau le plus bas possible. Par ailleurs, lorsque le coût de l'entreprise $A$ est inférieur à $\underline{v}_{B}$, dans la mesure où elle est certaine d'emporter le marché en soumettant moins que $\underline{v}_{B}$, sa stratégie de soumission à l'équilibre est définie de façon plus lâche.

Intéressons nous plus précisément à la soumission de l'entreprise $A$ lorsque $v_{A} \geq \underline{v}_{B}$ : $\frac{v_{A}+\alpha \underline{v}_{B}}{1+\alpha}$ et plus particulièrement à $\frac{v_{A}+\alpha \underline{v}_{B}}{1+\alpha}-v_{A}=\frac{\alpha\left(\underline{v}_{B}-v_{A}\right)}{1+\alpha}$, la différence entre le coût de réalisation des travaux d'entretien et la valeur de la soumission que nous désignerons sous le terme de biais. Celui-ci a toujours une valeur négative puisque l'entreprise $A$ soumet moins que $v_{A}$.

On constate, tout d'abord, que la valeur absolue de ce biais est croissante en $\alpha$. Plus l'entreprise $A$ détient une fraction importante de la société d'autoroute, plus elle tend à biaiser vers le bas le niveau de ses soumissions. En effet, soumettre moins que $v_{A}$ a un coût, ce coût étant le risque d'emporter le marché pour un prix inférieur au coût de réalisation. Mais ce coût est décroissant en $\alpha$. De plus, le gain de l'entreprise $A$ à biaiser son offre à la baisse est croissant avec $\alpha$. En effet, plus $\alpha$ est élevé, plus l'entreprise $A$ récupère une partie importante du profit supplémentaire qu'il crée en biaisant à la baisse sa soumission. Cela explique que la valeur absolue du biais de l'entreprise $A$ soit croissante en $\alpha$.

Par ailleurs, la valeur absolue du biais est aussi croissante en $v_{A}$. On saisira l'intuition de ce résultat en considérant une faible variation de la soumission de l'entreprise $A$. Soit un $\varepsilon$ strictement positif et arbitrairement petit, $\forall v_{A}$ tel que $v_{A}-\varepsilon \geq \underline{v}_{B}$, l'entreprise $A$, en biaisant sa soumission à la baisse de $\varepsilon$, court le risque d'emporter le contrat et de payer un prix compris entre $v_{A}$ et $v_{A}-\varepsilon$ soit, en valeur espérée, un coût de $\frac{\varepsilon^{2}}{2-2 \underline{v}_{B}}$, indépendant de $v_{A}$. En revanche, le gain à ce biais, $\alpha \frac{v_{A}-\varepsilon-\underline{v}_{B}}{1-\underline{v}_{B}} \varepsilon$, varie avec $v_{A}$. En effet, quand $v_{A}$ est élevé, proche de 1 , l'entreprise $A$ perd presque toujours l'appel d'offre. Par conséquent, elle diminue le prix payé à l'entreprise $B$ de $\varepsilon$ avec une probabilité proche de 1 . En revanche, lorsque $v_{A}$ est proche de $\underline{v}_{B}$, l'entreprise $B$ n'emporte presque jamais l'appel d'offre et le gain à biaiser devient très faible. Le coût à biaiser est constant et le gain croissant avec $v_{A}$. La valeur absolue du biais est alors croissante en $v_{A}$.

Corollaire 1 Lorsque l'entreprise A obtient la fraction $\alpha$ du capital de la société d'autoroute, l'allocation des travaux d'entretien n'est pas efficace. L'entreprise A emporte l'appel d'offre y compris dans des situations où l'entreprise $B$ pourrait réaliser les travaux pour un coût plus faible.

L'entreprise $A$ soumet moins que son coût à réaliser les travaux d'entretien alors que l'entreprise $B$ soumet son coût à les réaliser. Par conséquent, l'allocation des travaux 
d'entretien est inefficace avec une probabilité non nulle ${ }^{14}$. L'inefficacité est une conséquence de l'allocation d'une partie du capital de la société d'autoroute à l'entreprise $A$. Le corollaire 1 indique donc qu'un Etat soucieux d'efficacité devrait considérer avec précaution le fait de vendre une fraction du capital d'une société d'autoroute à une entreprise de travaux publics, et ceci sans même considérer le poids que cette entreprise peut faire peser sur les décisions de la société d'autoroute.

Corollaire 2 Si l'entreprise A obtient la fraction $\alpha$ du capital de la société d'autoroute, le prix espéré payé par la société d'autoroute pour la réalisation du chantier d'entretien est $\frac{4+\left(1+\underline{v}_{B}\right)\left(\underline{v}_{B}+6 \alpha+3 \alpha^{2}\right)}{6(1+\alpha)^{2}}$. Le prix espéré payé la société d'autoroute pour la réalisation des travaux d'entretien est strictement plus faible lorsque la société $A$ achète les actions en période 1 que lorsque la société $C$ les acquiert.

La baisse du coût de réalisation des travaux est une autre conséquence directe du biais de soumission de l'entreprise $A$. En soumettant moins que son coût à réaliser les travaux d'entretien, l'entreprise $A$ fait baisser le prix payé par la société d'autoroute. Pour la société d'autoroute, si elle ne se soucie pas d'efficacité, il est donc intéressant d'avoir parmi ses actionnaires un fournisseur potentiel. Cela lui permet de réduire le prix payé pour la réalisation du chantier d'entretien.

On peut élargir ce résultat qui ne s'exprime dans le corollaire qu'en espérance. En effet, il demeure vérifié (mais pas avec une baisse stricte) pour tout tirage de $v_{A}$ et $v_{B}$. Quelles que soient les valeurs de $v_{A}$ et $v_{B}$, le prix payé pour la réalisation des travaux quand l'entreprise $C$ obtient la participation au capital n'est jamais plus bas que lorsque l'entreprise $A$ obtient cette participation. En effet, l'entreprise $B$ soumet la même offre dans les deux cas et l'entreprise $A$ soumet une offre équivalente ou plus faible dans le second cas.

Ainsi, lorsqu'un fournisseur potentiel de la société d'autoroute -une entreprise de travaux publics- devient actionnaire de cette société d'autoroute, cela affecte l'appel d'offre organisé par la société d'autoroute pour l'entretien de ses voies. Cette observation s'applique alors même que nous faisons l'hypothèse conservatrice que l'entreprise actionnaire ne peut pas peser sur les décisions de la société d'autoroute. Sa seule présence au capital induit d'importantes distorsions : une modification du prix espéré payé pour la réalisation des travaux mais aussi une perte d'efficacité. Ceci s'explique par les transformations des stratégies de soumission de l'entreprise de travaux publics qui cherche à la fois à obtenir le contrat d'entretien pour le prix le plus favorable et à valoriser son investissement dans la société d'autoroute.

Troisième cas : l'entreprise $B$ achète en période 1 la fraction $\alpha$ du capital de la société d'autoroute.

\footnotetext{
${ }^{14}$ Elle est inefficace lorsque $v_{A}>v_{B} \geq v_{A}-\frac{\alpha}{1+\alpha}\left(v_{A}-\underline{v}_{B}\right)$, soit avec une probabilité égale à $\int_{\underline{v}_{B}}^{1} \frac{\alpha\left(v_{A}-\underline{v}_{B}\right)}{(1+\alpha)\left(1-\underline{v}_{B}\right)} d v_{A}=\frac{\alpha\left(1-\underline{v}_{B}\right)}{1+\alpha}$.
} 
La situation est inversée par rapport au cas précédemment étudié. L'entreprise $A$ a pour stratégie dominante de soumettre son coût à réaliser le chantier d'entretien. En revanche, l'entreprise $B$ est incitée à soumettre moins que son coût à le réaliser pour que l'entreprise $A$, en cas d'obtention du contrat, reçoive un paiement moindre. On retrouve un équilibre qui, dans sa forme, s'apparente à celui obtenu à la proposition 2.

Proposition 3 Si l'entreprise $B$ obtient la fraction $\alpha$ du capital de la société d'autoroute, en $t=3$, il existe un unique équilibre en stratégies non dominées. L'entreprise $A$ soumet $v_{A}$ et l'entreprise $B$ soumet $\frac{v_{B}}{1+\alpha}$.

L'allocation du chantier d'entretien n'est pas efficace. Il existe des situations où $v_{B}>v_{A}$ et l'entreprise $B$ emporte l'appel d'offre.

L'entreprise $B$ biaise sa soumission vers le bas pour réduire le prix payé dans le cas où l'entreprise $A$ emporterait l'appel d'offre. Les conséquences sont qualitativement proches de celles observées lorsque l'entreprise $A$ détient une fraction du capital de la société d'autoroute. Pour des motivations identiques, la valeur absolue du biais est croissante en $\alpha$ et $v_{B}$.

Corollaire 3 Si l'entreprise $B$ obtient la fraction $\alpha$ du capital de la société d'autoroute, le prix espéré payé par la société d'autoroute pour la réalisation du chantier d'entretien est $\frac{4+6 \alpha+3 \alpha^{2}+\underline{v}_{B}+\underline{v}_{B}^{2}}{6(1+\alpha)^{2}} \cdot 15$

Corollaire $4 \forall \underline{v}_{B}>0$, le prix espéré payé par la société d'autoroute pour la réalisation des travaux d'entretien est strictement plus faible lorsque la société $B$ achète les actions en période 1 que lorsque la société A les achète. La différence entre les prix espérés payés dans ces deux situations est égale à $\frac{\alpha(2+\alpha) \underline{v}_{B}}{2(1+\alpha)^{2}}$, strictement croissante en $\underline{v}_{B}$ et en $\alpha$.

Les corollaires 3 et 4 indiquent que, pour la société d'autoroute, non seulement il est intéressant d'avoir parmi ses fournisseurs un actionnaire mais qu'en plus, il est préférable que ce fournisseur soit celui qui dispose, a priori, de la structure de coût la moins favorable.

Pour mieux comprendre l'intuition de ce résultat, considérons des situations extrêmes. Soit $\underline{v}_{B}$ grand, proche de 1 . Si l'entreprise $A$ obtient les actions de la société d'autoroute, elle biaisera à la baisse sa soumission en seconde période. Cette baisse n'aura que peu d'effet sur le prix payé par la société d'autoroute puisque, avec une probabilité élevée, le prix est fixé par la soumission de l'entreprise $B$. En revanche, si l'entreprise $B$ achète, en $t=1$, les actions de la société d'autoroute et qu'en $t=3$, elle biaise à la baisse sa soumission, cela diminuera plus fortement le prix payé par la société d'autoroute dans la mesure où celui-ci est, le plus souvent, égal à la soumission

\footnotetext{
${ }^{15}$ On remarquera que, si $\underline{v}_{B}=0$, le prix espéré payé est égal à $\frac{4+6 \alpha+3 \alpha^{2}}{6(1+\alpha)^{2}}$ que l'entreprise $A$ ou l'entreprise $B$ obtienne les actions de la société d'autoroute.
} 
de l'entreprise $B$. Par conséquent, l'écart de prix payé entre les deux allocations des actions de la société d'autoroute est important. A l'opposé, lorsque $\underline{v}_{B}$ est proche de 0 , les entreprises $A$ et $B$ sont presque identiques et allouer les actions à une entreprise plutôt qu'à l'autre n'affecte que marginalement le prix payé pour les travaux d'entretien.

Il est ainsi plus intéressant de renforcer l'agressivité ${ }^{16}$ d'un fournisseur faible $(B)$, plutôt que d'un fournisseur fort $(A)^{17}$. La différence entre ces deux options s'accentue avec la faiblesse du premier. Cependant, cette baisse du prix payé par la société d'autoroute a un coût en terme d'efficacité.

Proposition 4 Lorsque l'entreprise A obtient les actions de la société d'autoroute en $t=1, P S E=\frac{\alpha^{2}}{6(1+\alpha)^{2}}\left(1+\underline{v}_{B}^{2}-2 \underline{v}_{B}\right)$. Lorsque l'entreprise $B$ obtient les actions de la société d'autoroute en $t=1, P S E=\frac{\alpha^{2}}{6(1+\alpha)^{2}}\left(1+\underline{v}_{B}^{2}+\underline{v}_{B}\right)$.

Corollaire 5 La perte de surplus espérée est plus élevée lorsque l'entreprise $B$ obtient les actions de la société d'autoroute en $t=1$ que lorsque l'entreprise A les obtient. Elle est même strictement plus élevée dans le cas $\underline{v}_{B}>0$.

Ainsi, la baisse du prix payé est plus forte lorsque l'entreprise $B$ achète les actions en $t=1$ mais la perte de surplus aussi.

La réduction du prix espéré payé pour les travaux d'entretien et la maximisation de l'efficacité allocative de ces travaux semblent donc être deux objectifs inconciliables. En effet, si en $t=1$, l'entreprise $C$ obtient les actions, les travaux d'entretien sont alloués de façon efficace mais le prix espéré payé pour la réalisation des travaux est maximal. A l'opposé, si l'entreprise $B$ obtient les actions, le prix espéré des travaux et l'efficacité allocative sont à leur minimum ${ }^{18}$. Enfin, si l'entreprise $A$ obtient les actions, efficacité allocative et prix espéré des travaux ont des valeurs intermédiaires. La préférence de l'Etat entre ces trois situations dépend de la taille de $(\theta-\alpha)$, la portion du capital de la société d'autoroute demeurant en possession de l'Etat, du coût de financement des fonds publics et de l'importance donnée à l'efficacité allocative. Nous analysons plus en détails, dans la section suivante, l'allocation de cette fraction du capital.

\section{La vente des actions}

Dans cette section, nous commençons par établir les éléments nécessaires à l'analyse, puis nous étudions l'allocation et le revenu obtenus avec les principaux formats

\footnotetext{
${ }^{16}$ Rendre une entreprise agressive dans un appel d'offre consiste à la pousser à faire des soumissions plus faibles.

${ }^{17}$ La notion de fournisseur faible ou fort se réfère à la structure de coûts plus ou moins favorable des entreprises en question.

${ }^{18} \mathrm{Il}$ s'agit d'un minimum par rapport aux deux autres situations possibles analysées.
} 
d'enchères, enfin, suivant une approche plus normative, nous proposons un mécanisme de vente des actions alternatif permettant à la fois de maximiser le revenu de l'Etat et d'assurer l'efficacité de l'allocation des travaux d'entretien.

Dans cette section, afin d'alléger l'exposition des résultats, nous supposerons que que $\underline{v}_{B}>0$ et indiquerons dans une remarque, en fin de section, les résultats obtenus dans le cas $\underline{v}_{B}=0$.

\subsection{Analyse préliminaire}

Dans le cadre traditionnel de l'étude des enchères, on suppose qu'un enchérisseur n'a pas de préférences concernant l'identité du vainqueur dans le cas où il perd l'enchère. Or, nous avons vu que les comportements des entreprises de travaux publics lors de l'appel d'offre en $t=3$ diffèrent suivant l'identité du vainqueur de la vente des actions en $t=1$. Ainsi, par exemple, pour l'entreprise $A$, il n'est pas équivalent que l'entreprise $B$ ou l'entreprice $C$ obtienne les actions de la société d'autoroute. L'entreprise $B$ sera plus agressive lors de l'appel d'offre dans le premier cas que dans le second cas. Les entreprises se soucient donc de l'allocation des actions en vente même lorsqu'elles perdent l'enchère. La vente des actions des sociétés d'autoroute rentrent alors dans le cadre théorique des enchères avec externalités allocatives telles que définies dans JEHIEL et AL [1996b] dont nous suivrons la méthodologie.

L'analyse d'une enchère avec externalités allocatives nécessite non seulement de connaître l'utilité des participants lorsqu'ils obtiennent le bien mais aussi leurs utilités pour toutes les allocations possibles du bien en vente. En effet, la valeur des actions pour une entreprise, dans ce contexte, ne peut plus être définie comme l'utilité espérée de cette l'entreprise lorsqu'elle obtient les actions. Elle se définira plutôt comme la différence d'utilité entre une situation où elle obtient ces actions et une situation où elles sont vendues à un concurrent, la différence en question étant fonction de l'identité de ce concurrent vainqueur (comme nous l'avons déjà exprimé, l'utilité espérée de l'agent $A$-tenant compte de l'appel d'offre pour les travaux d'entretien- n'est pas la même si l'entreprise $C$ ou si l'entreprise $B$ obtient les actions de la société d'autoroute).

C'est pourquoi nous avons besoin d'introduire la notation suivante : $U_{i}^{j}$ est l'espérance d'utilité de l'entreprise $i$ à la fin de la période 1 après que les actions ont été vendues à l'entreprise $j$. Cette espérance d'utilité ne tient pas compte des transferts liés à la vente des actions par l'Etat.

On notera que, dans la mesure où, au début comme à la fin de la période 1, l'information disponible est encore identique pour tous les acteurs (l'asymétrie n'apparaît qu'en période 2), l'enchère en période 1 se déroule en information symétrique entre tous les participants. Tous les $U_{i}^{j}$ sont connaissance commune en période 1.

Les valeurs des $U_{i}^{j}$ s'obtiennent à partir des fonctions de soumission d'équilibre 
identifiées dans la section précédente, en calculant les espérances d'obtenir la réalisation des travaux d'entretien en période 3 et l'espérance du prix payé pour la réalisation de ces travaux. Ainsi $U_{A}^{A}$ est égal à :

$$
\begin{aligned}
\underline{v}_{B}\left(\frac{-\underline{v}_{B}}{2}+(1-\alpha)\right. & \left.\frac{1+\underline{v}_{B}}{2}\right)+ \\
& \int_{\underline{v}_{B}}^{1}\left[-t+(1-\alpha)\left(\frac{\left.\left.1+\frac{t+\alpha \underline{v}_{B}}{1+\alpha}\right)\right]\left[\frac{1-\frac{t+\alpha \underline{v}_{B}}{1+\alpha}}{1-\underline{v}_{B}}\right] d t-}{2}\right.\right. \\
& \alpha \int_{\underline{v}_{B}}^{\frac{1+\alpha \underline{v}_{B}}{1+\alpha}}\left(1-t-\left(t-\underline{v}_{B}\right)\right)\left(\frac{t+\frac{1+\alpha \underline{v}_{B}}{1+\alpha}}{2}\right) \frac{1}{1-\underline{v}_{B}} d t+\alpha R N
\end{aligned}
$$

Le premier terme correspond aux cas où $v_{A} \in\left[0 ; \underline{v}_{B}\right]$, le second terme aux cas où $\left.\left.v_{A} \in\right] \underline{v}_{B} ; 1\right]$ et l'entreprise $A$ gagne l'appel d'offre et le troisième terme au cas où $\left.\left.v_{A} \in\right] \underline{v}_{B} ; 1\right]$ et l'entreprise $B$ gagne l'appel d'offre. Vu la lourdeur de ce type de formule, nous ne présenterons pas ici la valeur de tous les $U_{i}^{j}$.

Examinons, néanmoins, ce que nous pouvons dire sur les $U_{i}^{j}$, en commençant par les $U_{C}^{j}$.

On remarquera, tout d'abord, que $U_{C}^{A}=U_{C}^{B}=0$ puisque lorsque l'entreprise $C$ n'obtient pas d'actions de la société d'autoroute, elle ne réalise aucun profit, ne participant pas à l'appel d'offre. De plus, $U_{C}^{C}$ découle directement de l'espérance du prix atteint par l'appel d'offre lorsque l'entreprise $C$ achète les actions, soit $U_{C}^{C}=$ $\alpha\left(R N-\frac{1}{6}\left(4+\underline{v}_{B}+\underline{v}_{B}^{2}\right)\right)$.

Ces raisonnements simples ne s'appliquent pas pour les $U_{A}^{j}$ et $U_{B}^{j}$ puisque les entreprises $A$ et $B$, quelle que soit l'allocation des actions de la société d'autoroute à la période 1 , participent à l'appel d'offre en période 2. Or, nous avons vu, dans la section précédente, que l'issue de cet appel d'offre dépend de la structure capitalistique obtenue à la fin de la période 1 . Ainsi, sans calculer $U_{A}^{B}, U_{A}^{C}, U_{B}^{A}$ et $U_{B}^{C}$, on peut, a minima, affirmer que $U_{A}^{B} \neq U_{A}^{C}$ et $U_{B}^{A} \neq U_{B}^{C}$. En effet, comme nous l'indiquions dans la section précédente lorsque l'entreprise $B$ (resp : $A$ ) obtient les actions en $t=1$, elle est plus agressive lors de l'appel d'offre en $t=3$. Cela a un impact négatif sur l'utilité de l'entreprise $A$ (resp : $B$ ) qui préférerait que l'entreprise $C$ les obtienne.

Dès lors, il n'existe effectivement pas de valeur unique des actions pour les entreprises $A$ et $B$. Les prix qu'elles sont disposées à payer pour ces actions dépendent de leurs perceptions de l'alternative à leur obtention des actions. Si l'entreprise $A$ pense que, si elle n'obtient pas les actions, l'entreprise $B$ les obtiendra, elle sera disposée à payer pour les acheter au maximum $U_{A}^{A}-U_{A}^{B}$ alors que si elle pense que l'entreprise $C$ les obtiendrait dans ce cas, elle sera disposée à payer $U_{A}^{A}-U_{A}^{C}$.

Afin de poursuivre l'analyse de l'allocation des actions de façon plus approfondie dans les deux sous-sections suivantes, nous établissons quelques éléments de comparai- 
son entre les $U_{i}^{j}$ dans le lemme suivant ${ }^{19}$.

Lemme $1 \forall \underline{v}_{B}>0$ :

- $U_{B}^{B}-U_{B}^{C}>U_{C}^{C}$ et $U_{A}^{A}-U_{A}^{C}>U_{C}^{C}$.

- $U_{A}^{A}-U_{A}^{B}>U_{B}^{B}-U_{B}^{A}>U_{C}^{C}$.

La première partie du lemme indique que si les entreprises $A$ et $B$ pensent toutes les deux que, si elles n'obtiennent pas le bien, $C$ l'obtiendra, le montant que ces entreprises sont disposées à payer est plus élevé que le montant maximal que l'entreprise $C$ est disposée à payer. Après quelques calculs, on peut aussi montrer que $U_{B}^{B}-U_{B}^{C}>U_{A}^{A}-U_{A}^{C}$. Cela signifie que, par rapport à une situation où l'entreprise $C$ détiendrait les actions de la société d'autoroute, l'entreprise $B$ tire un surcroît d'utilité à obtenir ces actions plus élevé que l'entreprise $A$. Ce résultat tendrait à nous convaincre que les actions ont une valeur plus élevée pour l'entreprise $B$ que pour l'entreprise $A$. La seconde partie du lemme indique qu'une telle conclusion serait cependant hâtive.

En effet, celle-ci indique que si chacune des entreprises, $A$ et $B$, anticipe que si elle n'obtient pas les actions sa concurrente directe les obtiendra, alors l'entreprise $A$ est disposée à payer un prix plus élevé que l'entreprise $B$. Contrairement au point illustré précédemment, l'entreprise $B$ n'est plus disposée à payer plus que l'entreprise $A$.

La différence entre ces deux observations s'explique de la façon suivante. Dans la situation considérée pour la seconde partie du lemme, l'entreprise $A$ compare son profit espéré dans le cas où elle obtient les actions à celui dans le cas où l'entreprise $B$ les obtient. Or, nous avons vu dans la section précédente que l'entreprise $A$ souffre fortement des soumissions plus agressives de l'entreprise $B$ lorsque celle-ci détient les actions. $U_{A}^{B}$ a donc une valeur relativement faible ce qui augmente la valeur de $U_{A}^{A}-U_{A}^{B}$. Dans la section précédente, nous avions aussi observé que l'entreprise $B$ souffre moins fortement de la détention par l'entreprise $A$ des actions (que l'entreprise $A$ ne souffre de cette détention par l'entreprise $B) . U_{B}^{A}$ a donc, en termes relatifs, une valeur plus élevée ce qui réduit la valeur de $U_{B}^{B}-U_{B}^{A}$. Ces deux effets se cumulant, nous obtenons le résultat exprimé dans le lemme 1.

\subsection{Vente des actions au travers d'un format d'enchère stan- dard}

Nous nous intéressons ici au résultat qu'obtiendrait l'Etat s'il vendait les actions de la société d'autoroute au travers de formats d'enchères standards. Nous considérons

\footnotetext{
${ }^{19}$ Les preuves du lemme 1 figurent en annexe et s'appuient uniquement sur les parties qualitatives des corollaires 1, 2, 4 et 5. Le lemme 1 ne repose donc pas spécifiquement sur la forme des fonctions de distributions de $v_{A}$ et $v_{B}$ mais plus particulièrement sur l'asymétrie de ces fonctions de distribution, favorable à l'entreprise $A$ et les résultats qualitatifs qui en découlent.
} 
pour cela, l'enchère au premier et l'enchère au second prix ${ }^{20}$.

Nous avons présenté l'enchère au second prix dans la section 2. Le déroulement de l'enchère au premier prix est identique à celui de l'enchère au second prix. Il n'y a qu'une seule différence entre ces deux formats. Dans l'enchère au premier prix, lorsque l'acheteur ayant fait l'offre la plus élevée emporte l'enchère, il ne paye pas la seconde soumission la plus élevée mais un prix équivalent à sa propre soumission. Par ailleurs nous imposons la règle suivante pour résoudre les cas d'égalité dans l'enchère au premier prix. Si plus d'un enchérisseur propose la soumission la plus élevée, il est demandé à chacun de ces enchérisseurs de donner $\varepsilon$ ( $>0$ et arbitrairement petit) à un organisme extérieur. Si aucun des enchérisseurs concernés ne verse le montant $\varepsilon$, le bien est vendu avec une probabilité égale à chacun des enchérisseurs ayant fait la soumission la plus haute, au prix correspondant à cette soumission. Si au moins un enchérisseur accepte de verser le montant $\varepsilon$, le bien est vendu avec une probabilité égale à chacun des enchérisseurs ayant versé le montant $\varepsilon$ au prix correspondant à la soumission la plus élevée $e^{21}$.

Proposition 5 En $t=1$, que l'Etat utilise une enchère au premier prix ou au second prix, pour tout équilibre en stratégies non dominées, l'entreprise A obtient les actions pour un prix égal à $U_{B}^{B}-U_{B}^{A}$.

Le choix d'un format d'enchère parmi les formats d'enchères standards (enchère ascendante, au mieux disant...) n'affecte pas l'issue du processus d'allocation. Dans tous les cas, l'entreprise $A$ obtient les actions pour un prix égal à $U_{B}^{B}-U_{B}^{A}$, le prix pour lequel l'entreprise $B$ est indifférente entre obtenir les actions et que l'entreprise $A$ les obtienne.

Notons que cette allocation ne permet pas d'obtenir l'efficacité de l'appel d'offre portant sur la réalisation des travaux en $t=3$ puisque l'entreprise $C$ n'obtient pas les actions.

Néanmoins, dans la mesure où la privatisation des sociétés d'autoroute s'effectue principalement pour résoudre des problèmes de financement public, on peut considérer que, malgré le coût en terme d'efficacité en $t=3$, l'Etat choisira de vendre les actions à l'entreprise $A$ en $t=1$ puisque cela lui permet d'obtenir un revenu plus élevé que le maximum de ce qu'il pourrait obtenir de l'entreprise $C$ pour qu'elle achète les actions, soit $U_{C}^{C}$. En effet le lemme 1 indique que $U_{B}^{B}-U_{B}^{A}>U_{C}^{C}$. Nous prenons comme élément de comparaison une vente à l'entreprise $C$ puisque seule une telle vente permettrait de rétablir l'efficacité allocative en $t=3$.

\footnotetext{
${ }^{20}$ Qui sont strictement équivalentes, dans ce contexte, respectivement, à l'enchère ascendante et à l'enchère descendante.

${ }^{21}$ Cette règle spécifique pour résoudre les cas d'égalité est nécessaire à l'existence d'un équilibre (CF JACKSON et AL [2002]).
} 
Revenons un instant sur l'issue de l'enchère, l'allocation des actions à l'entreprise A. Autant il était prévisible que, à l'équilibre, une entreprise de travaux publics obtient les actions ${ }^{22}$ autant il n'était pas évident, a priori, que, à l'équilibre, l'entreprise $A$ emporte ces actions. En effet, dans la section précédente, nous avions montré que l'entreprise $B$ tire un parti plus fort de la détention des actions de la société d'autoroute que l'entreprise $A$. Elle modifie de façon plus importante ses soumissions lors de l'appel d'offre pour les travaux d'entretien. Néanmoins, l'entreprise $A$ est disposée à surenchérir sur les offres de l'entreprise $B$. Ceci, non pas parce que les actions de la société d'autoroute ont intrinsèquement une valeur plus élevée pour l'entreprise $A$ que pour l'entreprise $B$ mais parce que l'entreprise $A$ souffrirait fortement de la détention des actions par l'entreprise $B . U_{A}^{A}-U_{A}^{B}>U_{B}^{B}-U_{B}^{A}$ car $U_{A}^{B}$ faible comme nous l'avions expliqué en commentant le lemme 1.

\subsection{Définition d'un mécanisme optimal de vente des actions}

Après avoir décrit le revenu et l'allocation obtenu au travers des formats d'enchères standards, nous nous proposons de suivre une démarche plus normative et de suggérer un mécanisme de vente alternatif qui permet à la fois de rétablir l'efficacité lors de l'allocation des travaux d'entretien et de maximiser le revenu de l'Etat ${ }^{23}$.

Soit le mécanisme d'allocation M1 suivant :

Dans un premier temps, l'Etat demande à chacune des entreprises si elle souhaite participer ou non au mécanisme d'allocation. Celles-ci doivent répondre simultanément à cette demande.

Dans un second temps, l'Etat procède à l'allocation des actions et aux transferts avec les entreprises suivant les décisions de participation exprimées.

- Si toutes les entreprises décident de participer, l'Etat alloue les actions à l'entreprise $C$ et impose à l'entreprise $C$ de payer $U_{C}^{C}$, à l'entreprise $A$ de payer $U_{A}^{C}-U_{A}^{B}$ et à l'entreprise $B$ de payer $U_{B}^{C}-U_{B}^{A}$.

- Si l'entreprise $C$ décide de ne pas participer, les actions ne sont pas vendues et aucun transfert n'est réalisé.

- Si les entreprises $A$ et $C$ participent mais pas l'entreprise $B$, les actions sont vendues à l'entreprise $A$ au prix $U_{A}^{A}-U_{A}^{C}-\varepsilon$, l'entreprise $C$ recevant $\varepsilon$, avec $\varepsilon>0$ arbitrairement petit.

- Si les entreprises $B$ et $C$ participent mais pas l'entreprise $B$, les actions sont vendues à l'entreprise $A$ au prix $U_{B}^{B}-U_{B}^{C}-\varepsilon$, l'entreprise $C$ recevant $\varepsilon$, avec $\varepsilon>0$ arbitrairement petit.

\footnotetext{
${ }^{22}$ Puisque, comme nous l'avions montré dans la section précédente, les entreprises de travaux publics ont des motivations spécifiques pour acheter les actions.

${ }^{23}$ Ce mécanisme s'inspire des travaux de Jehiel et al (1996b).
} 
- Si seule l'entreprise $C$ participe, l'Etat lui vend les actions pour un prix égal à $U_{C}^{C}-\varepsilon$.

L'idée au coeur de ce mécanisme consiste à faire payer les entreprises $A$ et $B$ alors que les actions sont vendues à l'entreprise $C$. Les entreprises $A$ et $B$ acceptent de payer parce qu'elles savent que, en cas de refus de participer, les actions seront vendues à leurs concurrents directs, ce qui leur serait dommageable.

Proposition $6 \mathrm{Il}$ existe un unique équilibre en stratégies non dominées du mécanisme d'allocation M1. Tous les agents participent. L'entreprise $C$ obtient les actions et le revenu de l'Etat est égal à $U_{A}^{C}+U_{B}^{C}+U_{C}^{C}-U_{A}^{B}-U_{B}^{A}$. La vente des actions à l'entreprises $C$ permet une allocation efficace des travaux d'entretien en $t=3$.

Proposition $7 \forall \theta \in] 0 ; 1]$ et $\forall \alpha \in] 0 ; \theta]$, il n'existe pas de mécanisme d'allocation des actions en $t=1$ qui fournisse à l'Etat un revenu strictement supérieur à celui obtenu avec le mécanisme $M 1$.

Proposition 8 Si l'Etat utilise le mécanisme M1, son revenu espéré total incluant le prix de la vente des actions et les dividendes perçus de la société d'autoroute est égal à $U_{A}^{C}+U_{B}^{C}+U_{C}^{C}-U_{A}^{B}-U_{B}^{A}+(\theta-\alpha)\left(R N-\frac{1}{6}\left(4+\underline{v}_{B}+\underline{v}_{B}^{2}\right)\right)$. Il n'existe pas de mécanisme d'allocation en $t=1$ qui donne un revenu espéré total strictement supérieur.

Le mécanisme M1 assure donc à la fois le revenu le plus élevé possible pour l'Etat quelle que soit la façon dont le revenu est envisagé (en intégrant ou non les dividendes perçus de la société d'autoroute) et une efficacité de l'allocation des travaux d'entretien $^{24}$. En ce qui concerne les dividendes versés par la société d'autoroute, il serait plus favorable à l'Etat que l'entreprise $B$ obtienne les actions de la société d'autoroute. Néanmoins, si on considère l'ensemble du processus, le mécanisme M1 qui alloue ces actions à l'entreprise C maximise effectivement le revenu total de l'Etat. ${ }^{25}$

Ainsi, il est possible de concilier les objectifs de maximisation du revenu et d'efficacité, à condition de faire payer les entreprises pour leur éviter une allocation des actions qui leurs serait plus coûteuse.

\footnotetext{
${ }^{24}$ En première lecture, on pourrait interpréter ce résultat comme une simple application du Théorème de Coase. Ce n'est pas dans le cas. En effet, une partie des agents concernés, les actionnaires détenant la fraction $(1-\theta)$ des actions de la société d'autoroute restante ne participent pas au mécanisme. De plus, le mécanisme d'appel d'offre en $t=3$ est contraint.

${ }^{25}$ Dans l'ensemble de cette section, nous avons supposé que $\underline{v}_{B}>0$ afin de traiter spécifiquement le cas où les deux entreprises de travaux publics ne sont pas ex ante symétriques. Afin de compléter l'analyse, nous fournissons les résultats du cas $\underline{v}_{B}=0$. Dans ce cas, par symétrie, $U_{A}^{A}=U_{B}^{B}$ et $U_{A}^{B}=U_{B}^{A}$. Les enchères standards allouent alors les actions avec probabilité $1 / 2$ aux entreprises $A$ et $B$ pour un prix égal à $U_{A}^{A}-U_{A}^{B}\left(=U_{B}^{B}-U_{B}^{A}\right)$. Quant au mécanisme M1, il prend exactement la même forme que dans le cas $\underline{v}_{B}>0$, l'entreprise $C$ paye alors $U_{C}^{C}-U_{C}^{A}$ pour obtenir les actions et les deux autres entreprises payent $U_{A}^{C}-U_{A}^{B}\left(=U_{B}^{C}-U_{B}^{A}\right)$ pour éviter que le concurrent direct n'obtienne les actions. Le mécanisme M1 conserve ses propriétés d'efficacité et de maximisation du revenu.
} 


\section{Conclusion}

Nous avons montré que la vente des actions des sociétés d'autoroute à des entreprises de travaux publics peut obérer l'efficacité des appels d'offre même si l'Etat assure la complète transparence et l'absence de discrimination lors de ces appels d'offre. De plus, si ces actions sont allouées par un mécanisme de type vente au mieux disant (équivalent à un enchère au premier prix) ou une enchère ascendante, l'entreprise de travaux publics disposant, a priori, de la structure de coût la plus favorable, obtiendra les actions ce qui n'est pas forcément souhaitable du point de vue de la maximisation du revenu de l'Etat. Dans ce cadre, nous proposons un mécanisme d'allocation permettant à l'Etat d'obtenir un revenu maximum et de rétablir l'efficacité dans les appels d'offre organisés par la société d'autoroute.

Si on confronte les résultats découlant du modèle exposé aux faits, on observe que, lors des privatisations récentes de sociétés d'autoroute, dans deux cas sur trois (ASF et APRR), une entreprise de travaux publics de taille importante ${ }^{26}$ a effectivement obtenu les actions en vente, seule ou en association. L'allocation différente de la troisième société d'autoroute (SANEF) pourrait s'expliquer par une série d'éléments spécifiques : le retrait tardif de Bouygues dont le groupe se réoriente dans d'autres directions, les difficultés politiques associées à la vente simultanée à une seule entreprise de travaux publics des actions de deux sociétés d'autoroute, la forte volonté d'un opérateur étranger, Abertis, de s'introduire en France, même à un coût élevé. Notre analyse semblant être validée par la pratique, on pourra s'interroger sur l'utilisation d'une procédure d'allocation simple au mieux disant pour la vente des actions. Celle-ci n'a-t-elle pas été dommageable au revenu de l'Etat, en comparaison de ce qui aurait pu être obtenu en utilisant le mécanisme optimal identifié dans notre modèle ${ }^{27}$

Notons, enfin que les résultats obtenus avec le modèle présenté peuvent s'appliquer à une large palette de situations telles que : les délégations de gestion de parkings souterrains (à construire), les sociétés de capital-risque qui vendent une partie du capital de sociétés de hautes technologies à leurs clients ou leurs fournisseurs, les privatisations de sociétés de gestion d'aéroports au capital desquelles des entreprises de transport souhaiteraient prendre des participations capitalistiques (les biens rares que les sociétés de gestion d'aéroports ont à allouer sont les créneaux horaires et l'espace au sein de l'aéroport).

\footnotetext{
${ }^{26}$ Sans disposer d'informations sur les entreprises de travaux publics dans des situations plus ou moins favorables par rapport aux sociétés d'autoroute en vente, on supposera que les entreprises de travaux publics de taille importante disposent, a priori, de structures de coûts plus favorables.

${ }^{27} \mathrm{Il}$ convient, néanmoins, de noter que l'utilisation du mécanisme optimal n'était pas envisageable en raison de ses caractéristiques discriminatoires. Définir le mécanisme optimal non discriminatoire nécessiterait une définition économique des limites des contraintes de non discrimination.
} 


\section{Bibliographie}

Bati-Actu (2005) « Privatisation des sociétés d'autoroutes : L'Etat privilégie la logique financière $», 18$ juillet 2005.

Bulow, J., Huang, M., Klemperer, P. (1999) « Toeholds and Takeovers », Journal of Political Economy, 107, p. 427-454.

Burkart, M. (1995), «Initial Shareholding and Overbidding in Takeover Contests », Journal of Finance, 50, p. 1491-1515.

Caillaud, B., Jehiel, P. (1998) : «Collusion in Auction with Externalities », Rand Journal of Economics, 29, p. 680-702.

Cramton, P. (1997) : « The FCC Spectrum Auctions : An Early Assessment », Journal of Economics and Management Strategy, 6, p. 431-495.

Engelbrecht-Wiggans, R. (1994) : «Auctions with Price-Proportional Benefits to Bidders », Games and Economic Behavior, 6 , p. 339-346.

Ettinger, D. (2008), forthcoming, Annales d'Economie et de Statistique.

Jackson, M., Simon L. K., Swinkels J. M., Zame W. R. (2002) «quilibrium, Communication, Sharing Rules in Discontinuous Games of Incomplete Information », Econometrica, 70, p. 1711-1740.

Jehiel, P., Moldovanu, B. (1996a) « Strategic Non-Participation », RAND Journal of Economics, 27, p. 84-98.

Jehiel, P., Moldovanu, B., Stacchetti, E. (1996b) « How (Not) to Sell Nuclear Weapons », American Economic Review, 86, p. 814-829.

Le Monde (2005) « Le retrait de Bouygues complique la privatisation des autoroutes $», 30$ juillet 20005.

L'Express (2005) « 5 questions pour une privatisation », 15 août 2005.

Singh, R. (1998), «Takeover Bidding with Toeholds : the Case of the Owner's Curse», Review of Financial Studies, 11, p. 679-704. 


\section{A Preuves}

\section{A.1 Preuve de la Proposition 1}

Les entreprises $A$ et $B$ ne sont pas actionnaires de la société d'autoroute. Elles ne se soucient que de leurs probabilités de gagner l'appel d'offre et du prix qu'elles seront payées en cas d'obtention du marché. Dans ce cadre, soumettre son coût à réaliser les travaux est une stratégie dominante pour les raisons exposées dans le corps du papier. L'allocation est efficace puisque l'entreprise qui a le coût le plus bas obtient la réalisation du chantier.

Le coût espéré est égal à :

$$
\underline{v}_{B} \frac{\underline{v}_{B}+1}{2}+\int_{\underline{v}_{B}}^{1} \frac{1-t}{1-\underline{v}_{B}} \frac{1+t}{2} d t+\int_{\underline{v}_{B}}^{1} \frac{1}{1-\underline{v}_{B}} \frac{(1+t)(1-t)}{2} d t=\frac{1}{6}\left(4+\underline{v}_{B}+\underline{v}_{B}^{2}\right)
$$

Dans le membre de gauche de l'égalité, le premier terme recouvre les cas où $v_{A}<\underline{v}_{B}$, le second, $v_{B} \geq v_{A} \geq \underline{v}_{B}$ et le troisième, $v_{B}<v_{A}$.

C.Q.F.D.

\section{A.2 Preuve de la Proposition 2}

Pour les mêmes raisons que celles exprimées dans la preuve de la Proposition 1, l'entreprise $B$ a pour stratégie dominante de soumettre son coût à réaliser le chantier.

Calculons maintenant la meilleure réponse de l'entreprise $A$ en séparant les deux cas, $v_{A}<\underline{v}_{B}$ et $v_{A} \geq \underline{v}_{B}$.

$v_{A}<\underline{v}_{B}$. Quelle que soit la soumission de l'entreprise $B$ supérieure ou égale à $\underline{v}_{B}$, l'entreprise $A$ préférera soumettre moins et emporter l'appel d'offre. Toute meilleure réponse doit donc être inférieure à $\underline{v}_{B}$. Par ailleurs, pour que la stratégie de de l'entreprise $A$ ne soit pas dominée, il est nécessaire qu'elle ne soumette pas plus que son coût pour réaliser le chantier. Ces deux seules contraintes pèsent sur sa stratégie.

$v_{A} \geq \underline{v}_{B}$. On remarquera, tout d'abord, que l'entreprise $A$ peut se limiter à choisir une soumission optimale dans l'intervalle $\left[\underline{v}_{B} ; 1\right]$. En effet, en soumettant plus que 1 , elle ne gagnera jamais l'appel d'offre, elle peut obtenir ce même résultat en enchérissant 1. De plus, en enchérissant 1, elle réduit le prix payé par la société d'autoroute dont elle est actionnaire ce qui est dans son intérêt. De même, elle obtiendra la réalisation des travaux et pour un prix identique qu'elle enchérisse $\underline{v}_{B}$ ou moins que $\underline{v}_{B}$.

Soit l'utilité espérée de l'enchérisseur $A$ s'il soumet $s \in\left[\underline{v}_{B} ; 1\right]$ :

$$
-\alpha s \frac{s-\underline{v}_{B}}{1-\underline{v}_{B}}+\int_{s}^{1}\left(-v_{A}+(1-\alpha) t\right) \frac{1}{1-\underline{v}_{B}} d t+\alpha R N
$$

Le premier élément de cette formule recouvre l'utilité de l'agent A lorsqu'il perd l'appel d'offre et le second lorsqu'il emporte l'appel d'offre. Elle peut aussi s'écrire : 


$$
\frac{1}{1-\underline{v}_{B}}\left(\alpha \underline{v}_{B} s-v_{A}+\frac{1-\alpha}{2}+v_{A} s-(1+\alpha) \frac{s^{2}}{2}\right)+\alpha R N
$$

Maximiser cette expression, pour $s \in\left[\underline{v}_{B} ; 1\right]$ donne $: s=\frac{v_{A}+\alpha \underline{v}_{B}}{1+\alpha}$.

\section{A.3 Preuve du corollaire 2}

A l'équilibre l'espérance du prix payé peut s'écrire :

$$
\begin{aligned}
\frac{\left(1-\frac{1+\alpha \underline{v}_{B}}{1+\alpha}\right)}{1-\underline{v}_{B}} \frac{\left(1+\frac{1+\alpha \underline{v}_{B}}{1+\alpha}\right)}{2}+ & + \\
\int_{\underline{v}_{B}}^{\frac{1+\alpha \underline{v}_{B}}{1+\alpha}} \frac{t}{1-\underline{v}_{B}}\left(t(1+\alpha)-\alpha \underline{v}_{B}\right) d t+ & \int_{\underline{v}_{B}}^{1} \frac{v_{A}+\alpha \underline{v}_{B}}{1+\alpha}\left(\frac{v_{A}+\alpha \underline{v}_{B}}{1+\alpha}-\underline{v}_{B}\right) \frac{1}{1-\underline{v}_{B}} d v_{A}
\end{aligned}
$$

Le premier terme de cette expression recoupe les situations où $v_{B}$ est supérieure à la soumission de l'entreprise $A$ lorsque son coût à réaliser le chantier est 1 (dans ces cas, l'entreprise $A$ emporte toujours l'appel d'offre). Le second terme recoupe les situations où $v_{B}$ est inférieure à la soumission de l'entreprise $A$ lorsque son coût à réaliser le chantier est 1 et où l'entreprise $A$ emporte l'appel d'offre. Le troisième terme recoupe les situations où $v_{B}$ est inférieure à la soumission de l'entreprise $A$ lorsque son coût à réaliser le chantier est 1 et où l'entreprise $B$ emporte l'appel d'offre. Cette expression se simplifie en :

$$
\frac{4+\left(1+\underline{v}_{B}\right)\left(\underline{v}_{B}+6 \alpha+3 \alpha^{2}\right)}{6(1+\alpha)^{2}}
$$

C.Q.F.D.

\section{A.4 Preuve de la Proposition 3}

L'esprit de la preuve est identique à celui de la proposition 2. Pour des motivations symétriques, l'entreprise $A$ a pour stratégie dominante de soumettre son coût à réaliser le chantier et l'entreprise $B$, quel que soit son coût à réaliser le chantier, peut se limiter à choisir une soumission dans l'intervalle $[0 ; 1]$.

Soit l'utilité espérée de l'enchérisseur $B$ ayant un coût $v_{B}$ s'il soumet $s \in[0 ; 1]$ :

$$
(1-s)\left(-v_{B}+(1-\alpha) \frac{1+s}{2}\right)-\alpha s^{2}+\alpha R N
$$

La maximisation de cette expression donne $: s=\frac{v_{B}}{1+\alpha}$

C.Q.F.D. 


\section{A.5 Preuve du corollaire 3}

A l'équilibre l'espérance du prix payé peut s'écrire :

$$
\int_{\frac{1}{1+\alpha}}^{1} t d t+\int_{\frac{v_{B}}{1+\alpha}}^{\frac{1}{1+\alpha}} t \frac{(1+\alpha) t-\underline{v}_{B}}{1-\underline{v}_{B}} d t+\int_{\underline{v}_{B}}^{1} \frac{t}{1+\alpha} \frac{1}{1-\underline{v}_{B}} \frac{t}{1+\alpha} d t
$$

Le premier élément de cette formule est le prix espéré si l'entreprise $A$ a un coût supérieur à la soumission que l'entreprise $B$ soumet lorsque son coût est maximal, multiplié par la probabilité que $A$ ait un tel coût. Le second élément est le prix espéré lorsque $A$ a un coût inférieur à la soumission que l'entreprise $B$ soumet lorsque son coût est maximal mais que l'entreprise $B$ emporte l'appel d'offre, multiplié par la probabilité de cet événement. Le troisième élément est le prix espéré lorsque $A$ emporte l'appel d'offre, multiplié par la probabilité de cet événement.

Cette formule se simplifie et donne :

$$
\frac{4+6 \alpha+3 \alpha^{2}+\underline{v}_{B}+\underline{v}_{B}^{2}}{6(1+\alpha)^{2}}
$$

C.Q.F.D.

\section{A.6 Preuve de la Proposition 4}

Si l'entreprise $A$ obtient les actions, il y a perte de surplus lorsque $v_{A}>v_{B} \geq$ $\frac{v_{A}+\alpha \underline{v}_{B}}{1+\alpha}$. Pour $v_{A} \in\left[\underline{v}_{B} ; 1\right]^{28}$, cet événement se produit avec une probabilité égale à $\frac{v_{A}-\frac{v_{A}+\alpha \underline{v}_{B}}{1+\alpha}}{1-\underline{v}_{B}}=\frac{\alpha\left(v_{A}-\underline{v}_{B}\right)}{\left(1-\underline{v}_{B}\right)(1+\alpha)}$ et l'espérance de perte de surplus, conditionellement à cet événement, est alors de $\frac{v_{A}-\frac{v_{A}+\alpha \underline{v}_{B}}{1+\alpha}}{2}=\frac{\alpha\left(v_{A}-\underline{v}_{B}\right)}{2(1+\alpha)}$. La perte de surplus espérée peut alors s'écrire :

$$
\int_{\underline{v}_{B}}^{1} \frac{\alpha^{2}\left(v_{A}-\underline{v}_{B}\right)^{2}}{2(1+\alpha)^{2}\left(1-\underline{v}_{B}\right)} d v_{A}=\frac{\alpha^{2}\left(1-\underline{v}_{B}\right)^{2}}{6(1+\alpha)^{2}}
$$

Lorsque l'entreprise $B$ obtient les actions, il y a perte de surplus lorsque $v_{B}>v_{A} \geq$ $\frac{v_{B}}{1+\alpha}$. Quelle que soit la valeur de $v_{B}$, cet événement se produit avec probabilité $\frac{\alpha v_{B}}{1+\alpha}$ et l'espérance de perte de surplus, conditionellement à cet événement, est alors de $\frac{\alpha v_{B}}{2(1+\alpha)}$. La perte de surplus espérée peut alors s'écrire :

$$
\int_{\underline{v}_{B}}^{1} \frac{\alpha^{2} v_{B}^{2}}{2(1+\alpha)^{2}} \frac{1}{1-\underline{v}_{B}} d v_{B}=\frac{\alpha^{2}\left(1+\underline{v}_{B}+\underline{v}_{B}^{2}\right)}{6(1+\alpha)^{2}}
$$

C.Q.F.D.

\footnotetext{
${ }^{28} \mathrm{Si} v_{A}<\underline{v}_{B}$, cet événement est de probabilité nulle.
} 


\section{A.7 Preuve du Lemme 1}

\section{Première Partie :}

Les entreprises $A$ et $B$, dans le cas où elles obtiennent les actions, pourraient ne pas modifier leurs soumissions et enchérir leurs coûts à réaliser les travaux. Dans ce cas, l'utilité espérée des entreprises $A$ ou $B$ serait la même que dans le cas où l'entreprise $C$ obtient les actions à laquelle il faudrait ajouter $\alpha\left(R N-\frac{1}{6}\left(4+\underline{v}_{B}+\underline{v}_{B}^{2}\right)\right)=U_{C}^{C}$, le revenu espéré tiré de la possession des actions. D'où une espérance d'utilité pour l'entreprise $A$ égale à $U_{A}^{C}+U_{C}^{C}$ et pour l'entreprise $B: U_{B}^{C}+U_{C}^{C}$. Or, les entreprises $A$ et $B$ ne suivent pas ces stratégies (alors même que la stratégie de soumission de leurs adversaires dans l'appel d'offre est fixée et ne dépend pas de leurs stratégies de soumission). Nous avons vu précédemment que les entreprises $A$ et $B$ peuvent obtenir strictement plus en soumettant différemment. Il en découle donc que

$$
U_{A}^{A}>U_{A}^{C}+U_{C}^{C} \quad \text { et } \quad U_{B}^{B}>U_{B}^{C}+U_{C}^{C}
$$

Et par conséquent

$$
U_{A}^{A}-U_{A}^{C}>U_{C}^{C} \quad \text { et } \quad U_{B}^{B}-U_{B}^{C}>U_{C}^{C}
$$

Seconde partie :

Soit $E P^{i}$, l'espérance, considérée à la fin de la première période, du prix que la société d'autoroute obtiendra pour son appel d'offre en $t=3$, sachant que l'entreprise $i$ a obtenu les actions de la société d'autoroute en $t=1$. Soit $T^{i}$, le surplus total espéré, égal à la somme des utilités espérées des entreprises $A, B$ et $C$ et des actionnaires de la société d'autoroute (en dehors de la fraction $\alpha$ du capital vendue) à la fin de la première période, si l'entreprise $i$ obtient les actions. $T^{i}$ est égal à $U_{A}^{i}+U_{B}^{i}+U_{C}^{i}+(1-$ $\alpha)\left(R N-E P^{i}\right)$.

Des corollaires 1, 2, 4 et 5, on déduit que $E P^{C}>E P^{A}>E P^{B}$ et $T^{C}>T^{A}>T^{B}$. L'espérance du prix payé pour les travaux est plus faible si l'entreprise $A$ obtient les actions plutôt que l'entreprise $C$ et elle est encore plus faible si l'entreprise $B$ les obtient. Le classement du surplus total espéré est identique.

$T^{A}>T^{B}$ s'écrit :

$$
U_{A}^{A}+U_{B}^{A}+0+(1-\alpha)\left(R N-E P^{A}\right)>U_{A}^{B}+U_{B}^{B}+0+(1-\alpha)\left(R N-E P^{B}\right)
$$

D'où on déduit

$$
U_{A}^{A}-U_{A}^{B}>U_{B}^{B}-U_{B}^{A}+(1-\alpha)\left(E P^{A}-E P^{B}\right)
$$

Puisque $E P^{A}-E P^{B}>0$, on peut conclure que

$$
U_{A}^{A}-U_{A}^{B}>U_{B}^{B}-U_{B}^{A}
$$


D'autre part, nous savons que $U_{B}^{B}-U_{B}^{C}>U_{C}^{C}$ (voir la première partie de la preuve du lemme) et $U_{B}^{C}>U_{B}^{A}$ (l'entreprise $A$ fait des soumissions plus faibles dans l'appel d'offre en $t=3$ lorsqu'elle obtient les actions que lorsque l'entreprise $C$ les obtient), on en déduit que $U_{B}^{B}-U_{B}^{A}>U_{C}^{C}$.

C.Q.F.D.

\section{A.8 Preuve de la proposition 5}

\section{Enchère au second prix :}

Tout d'abord, observons que l'entreprise $C$ a pour stratégie dominante de soumettre $U_{C}^{C}$. Comme nous l'exposons dans la preuve de la proposition 1 , si le bien vendu a une valeur définie pour un acheteur, la stratégie dominante de cet acteur est de soumettre cette valeur.

Par ailleurs, on peut écarter la possibilité que l'entreprise $C$ gagne l'enchère. En effet, si l'entreprise $C$ gagne l'enchère avec une probabilité non nulle, cela signifie que le prix d'équilibre est inférieur ou égal à $U_{C}^{C}$ et que la soumission de l'entreprise $A$ (resp : $B$ )est inférieure ou égale à $U_{C}^{C}$. Or, nous avons vu, dans le lemme 1 , que $U_{A}^{A}-U_{A}^{C}>U_{C}^{C}$ (resp : $U_{B}^{B}-U_{B}^{C}>U_{C}^{C}$ ), par conséquent, les entreprises $A$ et $B$ préféreront toujours dévier et enchérir plus que $U_{C}^{C}$ afin d'obtenir les actions pour un prix égal à $U_{C}^{C}$ avec probabilité 1.

Puisque l'entreprise $C$ ne peut pas gagner l'enchère, l'entreprise $A$ a pour stratégie dominante de soumettre $U_{A}^{A}-U_{A}^{B}$, l'entreprise $B$ devenant son unique concurrent direct. De façon identique, l'entreprise $B$ a pour stratégie dominante d'enchérir $U_{B}^{B}-U_{B}^{A}$. Puisque $U_{A}^{A}-U_{A}^{B}>U_{B}^{B}-U_{B}^{A}$, l'entreprise $A$ emporte l'enchère et paye $U_{B}^{B}-U_{B}^{A} \geq$ $U_{B}^{B}-U_{B}^{C}>U_{C}^{C}$.

\section{Enchère au premier prix :}

Tout d'abord, notons que, pour tout équilibre en stratégie pure, au moins deux enchérisseurs soumettent la même enchère, la plus élevée. En effet, si ce n'était pas le cas, le vainqueur pourrait dévier de façon profitable en soumettant une valeur dans l'intervalle ouvert entre sa soumission et la seconde soumission la plus élevée.

Par ailleurs, pour l'entreprise $C$, soumettre plus que $U_{C}^{C}$ est une stratégie faiblement dominée puisqu'elle préfère perdre l'enchère à la gagner pour un tel prix.

Soit $p$ le prix d'équilibre. Si $p \leq U_{C}^{C}$, soit l'entreprise $A$ soit l'entreprise $B$ obtient les actions avec une probabilité inférieure à 1 . L'entreprise en question peut alors dévier de façon profitable en soumettant $p+\varepsilon$ avec $\varepsilon$ strictement positif et arbitrairement petit. Ceci implique que, à l'équilibre, $p>U_{C}^{C}$ et que seules les entreprises $A$ et $B$ soumettent $p$ à l'équilibre.

$p$ ne peut pas non plus être strictement supérieur à $U_{B}^{B}-U_{B}^{A}$ car soumettre plus que $U_{B}^{B}-U_{B}^{A}$ est une stratégie faiblement dominée pour l'entreprise $B$.

$p$ ne peut pas être strictement inférieur à $U_{B}^{B}-U_{B}^{A}$ car dans ce cas, au moins une 
des deux entreprises, $A$ ou $B$, obtiendrait les actions avec une probabilité inférieure à 1 et elle aurait intérêt à dévier en soumettant $p+\varepsilon$ avec $\varepsilon$ strictement positif et arbitrairement petit.

$p$ ne peut donc être égal qu'à $U_{B}^{B}-U_{B}^{A}$. Tout équilibre en stratégie non dominée prend alors la forme suivante. La soumission de l'entreprise $C$ est inférieure ou égale à $U_{C}^{C}$, les entreprises $A$ et $B$ soumettent $U_{B}^{B}-U_{B}^{A}$ et, au cours de la procédure pour départager qui suit, seule l'entreprise $A$ accepte de payer le $\varepsilon$ supplémentaire. C.Q.F.D.

\section{A.9 Preuve de la Proposition 6}

Pour démontrer la proposition, il suffit de prouver que toutes les entreprises choisissent de participer au mécanisme M1.

L'entreprise A. Comparons son utilité si elle participe et si elle ne participe pas pour les différents cas de figure. Si les entreprises B et C participent, en participant, l'entreprise A obtient une utilité égale à $U_{A}^{C}-\left(U_{A}^{C}-U_{A}^{B}\right)=U_{A}^{B}$ et, en ne participant pas, elle obtient une utilité égale à $U_{A}^{B}$ (puisque l'entreprise $\mathrm{B}$ obtient alors les actions). Si l'entreprise $\mathrm{C}$ ne participe pas, quelle que soit la décision de l'entreprise $\mathrm{A}$, son utilité est égale à $U_{A}^{C 29}$. Si l'entreprise $\mathrm{C}$ participe mais pas l'entreprise $\mathrm{B}$, en participant, l'entreprise A obtient une utilité égale à $U_{A}^{A}-\left(U_{A}^{A}-U_{A}^{C}-\varepsilon\right)=U_{A}^{C}+\varepsilon$, en ne participant pas, l'entreprise A obtient une utilité égale à $U_{A}^{C}$. Participer est alors une stratégie faiblement dominante pour l'entreprise A.

L'entreprise B. Comparons son utilité si elle participe et si elle ne participe pas pour les différents cas de figure. Si les entreprises A et C participent, en participant, l'entreprise B obtient une utilité égale à $U_{B}^{C}-\left(U_{B}^{C}-U_{B}^{A}\right)=U_{B}^{A}$ et, en ne participant pas, elle obtient une utilité égale à $U_{B}^{A}$ (puisque l'entreprise A obtient alors les actions). Si l'entreprise $\mathrm{C}$ ne participe pas, quelle que soit la décision de l'entreprise B, son utilité est égale à $U_{B}^{C}$. Si l'entreprise $\mathrm{C}$ participe mais pas l'entreprise $\mathrm{A}$, en participant, l'entreprise A obtient une utilité égale à $U_{B}^{B}-\left(U_{B}^{B}-U_{B}^{C}-\varepsilon\right)=U_{B}^{C}+\varepsilon$, en ne participant pas, l'entreprise $\mathrm{B}$ obtient une utilité égale à $U_{B}^{C}$. Participer est alors une stratégie faiblement dominante pour l'entreprise B.

L'entreprise C. Si elle ne participe pas, son utilité est égale à 0 . Si elle participe, suivant les décisions de participation des entreprises $\mathrm{B}$ et $\mathrm{C}$, son utilité est égale à 0 ou $\varepsilon>0$. Participer est alors une stratégie faiblement dominante pour l'entreprise C. C.Q.F.D.

\section{A.10 Preuve de la Proposition 7}

Soit une allocation qui donne les actions à l'entreprise $A$.

\footnotetext{
${ }^{29}$ Puisqu'en $t=3$, que l'entreprise $\mathrm{C}$ obtienne les actions ou qu'elles ne soient pas allouées, la situation est identique pour l'entreprise A
} 
L'Etat pourra extraire au maximum $U_{A}^{A}-U_{A}^{B}$ de l'entreprise $A$ dans le cas où il réalise cette allocation. En effet, si l'entreprise $A$ n'accepte pas ce mécanisme d'allocation, il peut la menacer au pire d'allouer le bien à l'entreprise $B$, ce qui lui donnerait une utilité égale à $U_{A}^{B}$. Or, en lui faisant payer $U_{A}^{A}-U_{A}^{B}$ pour les actions, il ramène son utilité à $U_{A}^{B}$, l'entreprise $A$ n'accepterait donc pas de payer plus. Elle préférerait ne pas participer et que les actions soient allouées à l'entreprise $B$. L'Etat ne pourra rien extraire de l'entreprise $B$ s'il réalise cette allocation car il ne peut pas menacer l'entreprise $B$ de réaliser une allocation plus défavorable que celle-ci, si elle ne participe pas car cette allocation est la plus défavorable pour l'entreprise $B$. Il ne pourra rien extraire de l'entreprise $C$ qui obtient une utilité nulle dans tous les cas où elle n'obtient pas les actions. Son revenu maximal est donc égal à $U_{A}^{A}-U_{A}^{B}$.

Avec exactement, le même type d'arguments, on peut montrer que l'Etat ne peut pas obtenir un revenu supérieur à $U_{B}^{B}-U_{B}^{A}$ s'il vend les actions à l'entreprise $B$.

Si l'Etat vend les actions à l'entreprise $C$. Celle-ci sera au maximum disposée à payer $U_{C}^{C}$, l'entreprise $A$ sera disposée à payer au maximum $U_{A}^{C}-U_{A}^{B}$ (si on la menace en cas de refus de payer d'allouer les actions à l'entreprise $B$ ) et l'entreprise $B$ sera disposée à payer au maximum $U_{B}^{C}-U_{B}^{A}$ (si on la menace en cas de refus de payer d'allouer les actions à l'entreprise $A$ ). Soit au total $U_{C}^{C}+U_{A}^{C}+U_{B}^{C}-U_{A}^{B}-U_{B}^{A}$.

Prouvons maintenant que : $U_{C}^{C}+U_{A}^{C}+U_{B}^{C}-U_{A}^{B}-U_{B}^{A}>U_{A}^{A}-U_{A}^{B}$ et $U_{C}^{C}+U_{A}^{C}+$ $U_{B}^{C}-U_{A}^{B}-U_{B}^{A}>U_{B}^{B}-U_{B}^{A}$.

Notons tout d'abord que la seconde partie du lemme 1 établissant que $U_{A}^{A}-U_{A}^{B}>$ $U_{B}^{B}-U_{B}^{A}$, il ne nous est nécessaire que de prouver que $U_{C}^{C}+U_{A}^{C}+U_{B}^{C}-U_{A}^{B}-U_{B}^{A}>$ $U_{A}^{A}-U_{A}^{B}$. Or nous savons que $T^{C}>T^{A}$ et $E P^{A}>E P^{C}$ (voir preuve du du lemme 1), d'où :

$$
\begin{array}{r}
T^{C}-T^{A}+(1-\alpha)\left(E P^{A}-E P^{C}\right)>0 \\
T^{C}-(1-\alpha) E P^{C}-T^{A}+(1-\alpha) E P^{A}>0 \\
U_{C}^{C}+U_{A}^{C}+U_{B}^{C}-U_{B}^{A}-U_{A}^{A}>0 \\
U_{C}^{C}+U_{A}^{C}+U_{B}^{C}-U_{A}^{B}-U_{B}^{A}>U_{A}^{A}-U_{A}^{B}
\end{array}
$$

Il n'est donc pas possible d'obtenir un revenu supérieur à $U_{C}^{C}+U_{A}^{C}+U_{B}^{C}-U_{A}^{B}-U_{B}^{A}$ en allouant les actions que ce soit à l'entreprise $A$, à l'entreprise $B$ ou à l'entreprise $C$.

De plus, un mécanisme d'allocation qui allouerait avec une probabilité non nulle les actions à plus d'une entreprise ne pourrait fournir un revenu supérieur à une combinaison convexe de $U_{A}^{A}-U_{A}^{B}, U_{B}^{B}-U_{B}^{A}$ et $U_{C}^{C}+U_{A}^{C}+U_{B}^{C}-U_{A}^{B}-U_{B}^{A}$, ce qui ne peut être supérieure à $U_{C}^{C}+U_{A}^{C}+U_{B}^{C}-U_{A}^{B}-U_{B}^{A}$.

C.Q.F.D. 


\section{A.11 Preuve de la Proposition 8}

Comme nous l'avons montré dans la preuve de la proposition 7, l'Etat ne peut pas obtenir plus que $U_{A}^{A}-U_{A}^{B}$ en vendant les actions à l'entreprise $A$ en $t=1$. En incluant les dividendes versés en $t=4$, cela donne un revenu maximum total égal à $U_{A}^{A}-U_{A}^{B}-(\theta-\alpha)\left(R N-E P^{A}\right)$.

De la même façon, en vendant les actions à l'entreprise $B$ en $t=1$, le revenu maximal total est égal à $U_{B}^{B}-U_{B}^{A}-(\theta-\alpha)\left(R N-E P^{B}\right)$ et en les vendant à l'entreprise $C$ en $t=1: U_{C}^{C}+U_{A}^{C}+U_{B}^{C}-U_{A}^{B}-U_{B}^{A}+(\theta-\alpha)\left(R N-E P^{C}\right)=U_{C}^{C}+U_{A}^{C}+U_{B}^{C}-$ $U_{A}^{B}-U_{B}^{A}+(\theta-\alpha)\left(R N-\frac{1}{6}\left(4+\underline{v}_{B}+\underline{v}_{B}^{2}\right)\right)$.

Comme pour la preuve de la proposition 7 , il faut alors montrer que

$$
U_{C}^{C}+U_{A}^{C}+U_{B}^{C}-U_{A}^{B}-U_{B}^{A}+(\theta-\alpha)\left(R N-E P^{C}\right)>U_{A}^{A}-U_{A}^{B}-(\theta-\alpha)\left(R N-E P^{A}\right)
$$

et

$$
U_{C}^{C}+U_{A}^{C}+U_{B}^{C}-U_{A}^{B}-U_{B}^{A}+(\theta-\alpha)\left(R N-E P^{C}\right)>U_{B}^{B}-U_{B}^{C}-(\theta-\alpha)\left(R N-E P^{B}\right)
$$

Commençons par montrer que la première inéquation est vérifiée. Nous savons que $T^{C}>T^{A}$ et $E P^{A}>E P^{C}$ (voir preuves précédentes), d'où :

$$
\begin{array}{r}
T^{C}-T^{A}+(1-\theta)\left(E P^{A}-E P^{C}\right)>0 \\
T^{C}-(1-\alpha) E P^{C}-\left(T^{A}-(1-\alpha) E P^{A}\right)+(\theta-\alpha)\left(E P^{C}-E P^{A}\right)>0 \\
U_{A}^{C}+U_{B}^{C}+U_{C}^{C}-U_{B}^{A}-U_{A}^{A}+(\theta-\alpha)\left(E P^{C}-E P^{A}\right)>0 \\
U_{C}^{C}+U_{A}^{C}+U_{B}^{C}-U_{A}^{B}-U_{B}^{A}+(\theta-\alpha)\left(R N-E P^{C}\right) \\
>U_{A}^{A}-U_{A}^{B}-(\theta-\alpha)\left(R N-E P^{A}\right)
\end{array}
$$

La seconde inéquation se démontre de façon strictement identique en partant des résultats connus : $T^{C}>T^{B}$ et $E P^{B}>E P^{C}$

C.Q.F.D. 